\title{
Thermal Conductivity of Carbon Nanotube Composite Films
}

Quoc Ngo ${ }^{1,2}$, Brett A. Cruden ${ }^{2}$, Alan M. Cassell ${ }^{2}$, Megan D. Walker ${ }^{2}$, Qi Ye ${ }^{2}$, Jessica E. Koehne $^{2}$, M. Meyyappan ${ }^{2}$, Jun $\mathrm{Li}^{2^{*}}$, and Cary Y. Yang ${ }^{1}$

${ }^{1}$ Center for Nanostructures, Santa Clara University, $500 \mathrm{El}$ Camino Real

Santa Clara, CA 95050, USA

${ }^{2}$ Center for Nanotechnology, NASA Ames Research Center

Moffett Field, CA, 94035, USA

\begin{abstract}
State-of-the-art ICs for microprocessors routinely dissipate power densities on the order of $50 \mathrm{~W} / \mathrm{cm}^{2}$. This large power is due to the localized heating of ICs operating at high frequencies, and must be managed for future high-frequency microelectronic applications. Our approach involves finding new and efficient thermally conductive materials. Exploiting carbon nanotube (CNT) films and composites for their superior axial thermal conductance properties has the potential for such an application requiring efficient heat transfer. In this work, we present thermal contact resistance measurement results for CNT and CNT-Cu composite films. It is shown that Cü-filled CNT arrays enhance thermal conductance when compared to as-grown CNT arrays. Furthermore, the CNT-Cu composite material provides a mechanically robust alternative to current IC packaging technology.
\end{abstract}

\section{INTRODUCTION}

As progress continues in the ultra-large-scale-integration (ULSI) of integrated circuits, microelectronic components including transistors, and more prominently interconnects, have become increasingly more dense and compact. The consequence of increased component density manifests itself in the form of locally high power consumption. An alarming rise in power density with respect to each advancing technology generation has been observed in mainstream microprocessor technologies [1]. The need for addressing this problem is imperative for next-generation IC packaging technology. One potential solution is to find new packaging materials, such as CNTs, that exhibit high thermal conductivity along the axial direction. For a discrete multiwalled nanotube (MWNT), the thermal conductivity is expected to surpass 3000 $\mathrm{Wm}^{-1} \mathrm{~K}^{-1}$ along the tube axis [2]. Through the use of DC-biased, plasma-enhanced chemical vapor deposition (PECVD), as demonstrated in [3], we can fabricate vertically aligned MWNT arrays on silicon wafers of $\sim 500 \mu \mathrm{m}$ thickness and demonstrate their possible application as a heat-sink device, conducting large amounts of heat away from a localized area, such as in critical "hot spots" in ICs. This work focuses on demonstrating that CNT and CNT-Cu composite films are mechanically robust, efficient thermal conductors. $\mathrm{Cu}$ serves as a filler material to improve the mechanical stability of the CNT array and to serve as a lateral heat spreader. Our data shows that the structures fabricated in this study are completely reusable, unlike eutectic bonding techniques currently used in packaging technology, which is particularly important for instrument cooling in space applications.

\footnotetext{
• corresponding author: jli@mail.arc.nasa.gov
} 


\section{EXPERIMENT}

\section{Description of apparatus}

An apparatus consisting of two copper blocks, four resistive cartridge heaters (not shown) embedded in the upper block, and a cooling bath was used to measure the thermal resistance of a given material (figure 1). The upper copper block is surrounded by insulation to minimize heat loss to the ambient, with the exception of the one square inch section designed to contact the material to be measured. The clamping pressure on the sample is controlled by pneumatically manipulating the upper block. Heat is delivered to the system by applying a constant power to the resistive heaters. The steady state temperature difference $\left(\Delta T=T_{B}-T_{C}\right)$ between the two blocks (and consequently, the sample) was measured. From this data, the thermal resistance of the sample is calculated, as shown in equation (1), where $\mathrm{Q}$ is the total power in (in W), $\mathrm{A}$ is the sample area, $C_{L}$ is the constant heat transfer coefficient and $T_{B}, T_{C}$, and $T_{\text {amb }}$ represent the temperature of the upper block, the chilled lower block $\left(20^{\circ} \mathrm{C}\right)$, and the ambient environment, respectively. $C_{\mathrm{L}}$ is used to estimate the heat loss to the ambient in this measurement configuration and is determined by placing a thick insulator between the two blocks and measuring the steady state $\Delta \mathrm{T}$ at a variety of constant powers. This analysis yields a constant heat transfer coefficient of $0.0939 \mathrm{~W} / \mathrm{K}$, representing the heat loss (in W) per Kelvin to the ambient, which is factored into the final determination of the measured thermal resistance

$$
R=\frac{A\left(T_{B}-T_{C}\right)}{Q-C_{L}\left(T_{B}-T_{a m b}\right)}
$$

It should be noted, however, that the dominant thermal resistance mechanism in this measurement configuration is that of the contact interfaces between the sample and the copper blocks. To minimize this contact resistance, two steps were taken: 1) polishing both copper blocks to reduce the effect of surface roughness and 2) making use of a high thermally conductive, conformal material, Microfaze A6 (AOS Thermal Compounds, LLC) to reduce contact resistance on the backside of a silicon wafer, the substrate on which the investigated films were fabricated.

\section{Sample preparation}

Carbon nanotubes were synthesized using the procedure and reactor conditions detailed in [3]. A layer of chromium (2000 $\AA$ ) was used as an adhesion layer for the thin layer of nickel catalyst used for the MWNT array growth. The resulting as-grown tubes are shown in figure 2. Using scanning electron microscope (SEM) data, we estimate the length of the tubes to be $\sim 7.5 \mu \mathrm{m}$. Following nanotube synthesis, copper filling between individual MWNTs (hereafter known as nanotube trenches) was accomplished through electrodeposition. In a three-electrode 

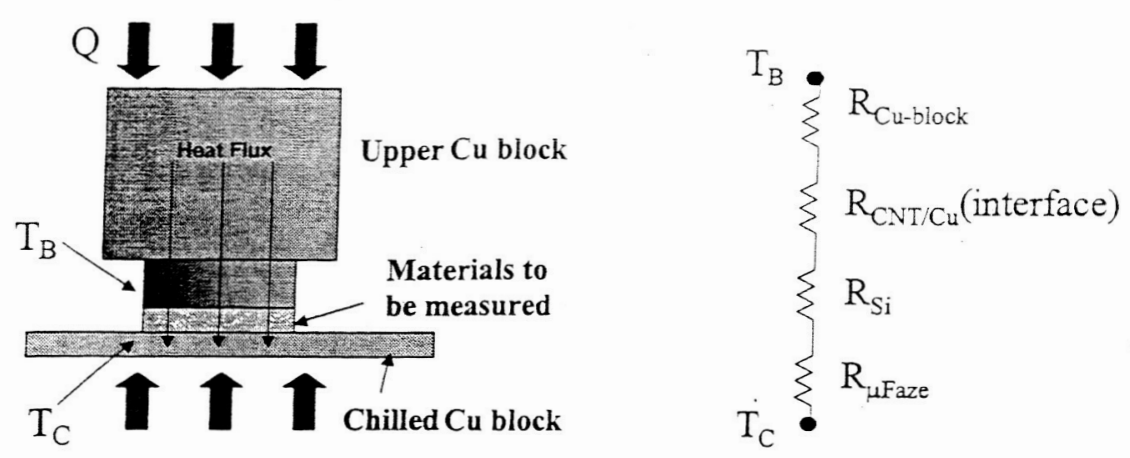

Figure 1. Apparatus used for thermal resistance measurement and equivalent thermal resistance model. $\mathrm{T}_{\mathrm{B}}$ is the temperature of the block, measured using an embedded thermocouple. $\mathrm{T}_{\mathrm{C}}$ is the temperature of the chilled lower copper block $\left(20^{\circ} \mathrm{C}\right)$.

setup with the MWNT sample as the working electrode, a Saturated Calomel Electrode (SCE) as the reference electrode, and a one square inch platinum foil as the counter electrode (CE), set in parallel with the MWNT sample. Both the Cr substrate and MWNTs serve as electrodes during the electrodeposition. Various additives were added in the solution to achieve optimum gap filling into the high-aspect-ratio forest-like MWNT arrays. The recipe of the electrolyte solution used in this study is based on the methodology reported for deep-trench filling of $\mathrm{Cu}$ interconnects for damascene processes [4]. Typically, the $\mathrm{Cu}$ was deposited at -0.20 to $-0.30 \mathrm{~V}$ (vs. SCE) at a deposition rate of $\sim 430 \mathrm{~nm} / \mathrm{min}$. The resulting CNT-Cu composite material has $\sim 30 \%$ submicron voids as shown in figure 3 .

\section{RESULTS AND DISCUSSION}

To summarize the structure used, figure 1 includes the equivalent thermal resistance model for the CNT-Cu composite sample. The resistance of the CNT-Cu composite can be obtained by de-embedding the thermal resistance contribution of the copper block $\left(\mathrm{R}_{\mathrm{Cu} \text {-block }}\right)$, silicon wafer $\left(\mathrm{R}_{S i}\right)$, and the Microfaze material $\left(\mathrm{R}_{\mu \mathrm{Faze}}\right)$. The thermal resistance of the copper block, $R_{\text {Cu-block, }}$ must be taken into account due to the placement of the thermocouple (approximately 1.3 inches from the copper block surface). $\mathrm{R}_{\text {Cu-block }}$ for this configuration is determined as $0.83 \mathrm{~cm}^{2} \mathrm{~K} / \mathrm{W}$ based on bulk copper properties. To summarize, we can determine the resistance of the $\mathrm{CNT}-\mathrm{Cu}$ composite film by equation (2).

$R_{C N T-C u}=R_{\text {total }}-R_{C u-b l o c k}-R_{S i}-R_{\mu \text { Faze }}$

$\mathrm{R}_{\mu \mathrm{Faze}}$ is determined using two control measurements. The first measurement involves measuring the thermal resistance of a piece of silicon with Microfaze on the backside of the wafer, resulting in $\mathrm{R}_{\text {control, } 1}=\mathrm{R}_{\text {Cu-block }}+\mathrm{R}_{\text {block-Si }}+\mathrm{R}_{\mu \mathrm{Faze}}$, where $\mathrm{R}_{\text {block-Si }}$ is the interface resistance between the copper block and silicon wafer. The second resistance measurement involves a piece of double-sided polished silicon, resulting in $\mathrm{R}_{\text {control, } 2}=2 \mathrm{R}_{\text {block-Si}}+\mathrm{R}_{\mathrm{Si}}$. Assuming both Si$\mathrm{Cu}$ interfaces in the second control measurement are similar, we can divide this value in half and use the simple relation in equation 3 .

$R_{\mu F a z e}=R_{\text {control, } 1}-R_{\text {Cu-block }}-0.5\left(R_{\text {control }, 2}-R_{S i}\right)$ 
The intrinsic silicon contribution to the thermal resistance in equations (2) and (3) can effectively be neglected. For the $500 \mu \mathrm{m}$ thick silicon wafer used in this study, the intrinsic silicon thermal resistance can be calculated as $0.034 \mathrm{~cm}^{2} \mathrm{~K}$ W, which proves to be two orders of magnitude less than the final measured values of the CNT-Cu sample. One caveat to this analysis is in regards to the thermal resistance of Microfaze with respect to the amount of power applied to the upper block. The thermal resistance of the first control sample decreases approximately exponentially for increasing powers (hence different temperature gradients), but can be corrected for in the final analysis as will be demonstrated. The double-sided silicon sample shows no such power dependence, exhibiting a constant resistance of $11.10 \mathrm{~cm}^{2} \mathrm{~K} / \mathrm{W}$, resulting in $5.55 \mathrm{~cm}^{2} \mathrm{~K} / \mathrm{W}$ per silicon interface. Subtracting the silicon resistance, which is constant with respect to power, we can also determine $R_{\mu F a z e}$ at different powers. The power dependence of the Microfaze is captured in figure $4(\mathrm{a})$.

Now that the power dependence of the Microfaze material is quantified, we proceed with the analysis of the CNT/Si/Microfaze and CNT-Cu/Si/Microfaze stacks. From the previous discussion, we can expect these samples also to exhibit the same power dependence, which indeed is the case and is clearly seen in figure 4(b). Combining the power dependence with the measurements in figure 4(b), we summarize the values of measured thermal resistance in table I.
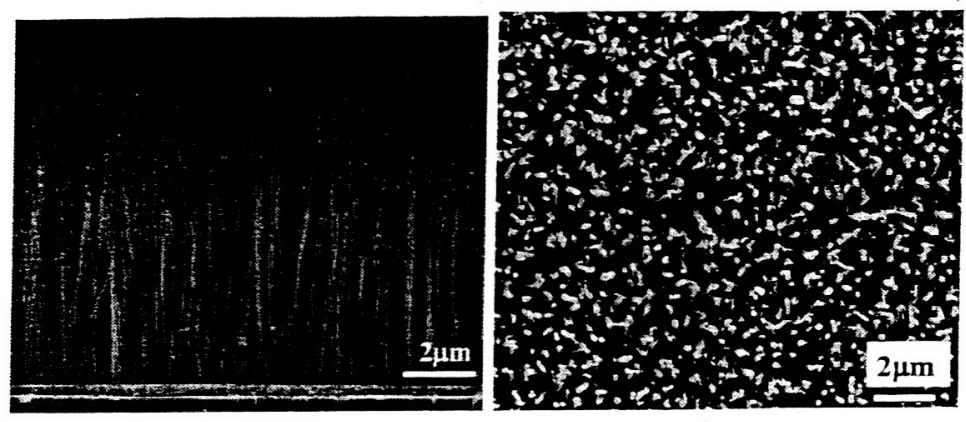

Figure 2. SEM micrographs (cross-section and top-down) of as-grown MWNT film. Length of nanotubes is estimated as $\sim 7.5 \mu \mathrm{m}$.
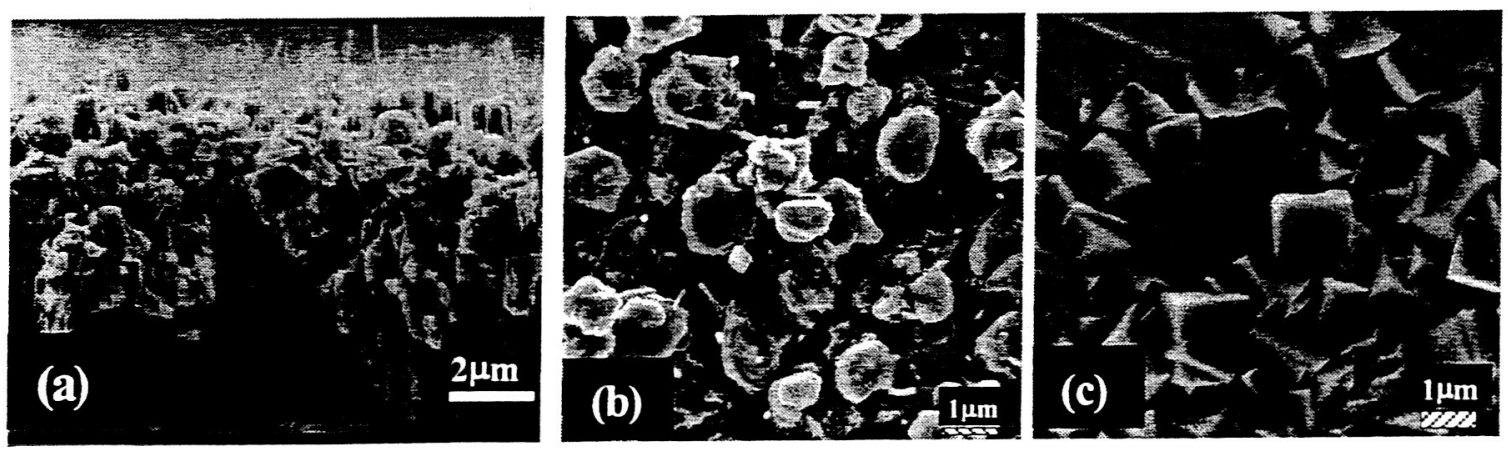

Figure 3. SEM micrographs (a) cross-section (b) top-down of CNT-Cu composite film after filling nanotube trenches by copper electrodeposition using recipe in [4]. (c) shows a top-down view of copper electrodeposition with low additive concentration. 
All measurements were performed at similar clamp pressures, $\sim 6.8$ psi. Errors causing the standard deviation in the measurements can be attributed to two main factors: 1) variations in contact area due to varying CNT length distribution (see fig. 2) and 2) variations in measurement of total power, $\Delta \mathrm{T}$, and ambient temperature loss. However, even at the upper bounds of the measured thermal resistance values for the $\mathrm{CNT}-\mathrm{Cu}$ composite films, this worst-case scenario represents values that are on the order of the thermal budgets for a variety of commercial microprocessor systems [1].

It is noted that the Cu deposited in the MWNT array used in this study was not a solid film. Instead, it forms a porous film with $\sim 70 \% \mathrm{Cu}$ and $\mathrm{CNTs}$ and $\sim 30 \%$ voids. This increases the mechanical strength so that the sample can be repeatedly measured under different clamping pressures. In addition, it leaves space such that the composite film can be deformed to make maximal contact with the hot surface. However, studies conducted on the buckling force of discrete MWNTs [5-7] demonstrate the tremendous amount of force that these structures can withstand. Based on this analysis, we can speculate that nanotubes will not buckle under the force applied in this study, roughly two orders of magnitude less than the calculated CNT buckling force. This speculation is confirmed by SEM characterization before and after the thermal resistance measurement, seen in figure 5, showing no effect on the CNT-Cu composite after compressive stress. Measurements conducted with higher clamp pressures are underway to confirm the buckling force and optimize thermal conductance. Future work will be focused on increasing the exposed length of CNTs to obtain a lower buckling force. The exposed CNTs serve as the thermal interface material, while $\mathrm{Cu}$ is only filled at the bottom portion of the structure to serve as both a mechanical anchor and lateral heat spreader.

\section{CONCLUSION}

This work demonstrates the fundamental usefulness of CNTs and CNT-Cu composite films as efficient heat conductors. Our study confirms that these novel films can accomplish effective heat conduction by increasing contact area. In addition, it has been shown that CNTs provide the added benefit of high mechanical stability and reusability. Further characterization and investigation are in progress to integrate such a process into packaging process flows.

\section{ACKNOWLEDGEMENTS}

MDW is an undergraduate intern at NASA Ames Research Center from the University of Oklahoma, Tulsa. BAC and AMC are also with the University Affiliated Research Center at University of California, Santa Cruz. QY is with ELORET. NASA contract number NAS203144 supports both UARC and ELORET.

Table I. Thermal Resistance measurement summary

\begin{tabular}{|l|l|}
\hline Material & Thermal Resistance $\left(\mathrm{cm}^{2} \mathbf{K} / \mathbf{W}\right) \pm$ STDEV \\
\hline CNT film & $2.42 \pm 0.33$ \\
\hline CNT-Cu composite film (\#1) & $1.05 \pm 0.22$ \\
\hline CNT-Cu composite film (\#2) & $0.96 \pm 0.13$ \\
\hline Bare double-sided silicon & $11.10 \pm 0.65$ \\
\hline
\end{tabular}



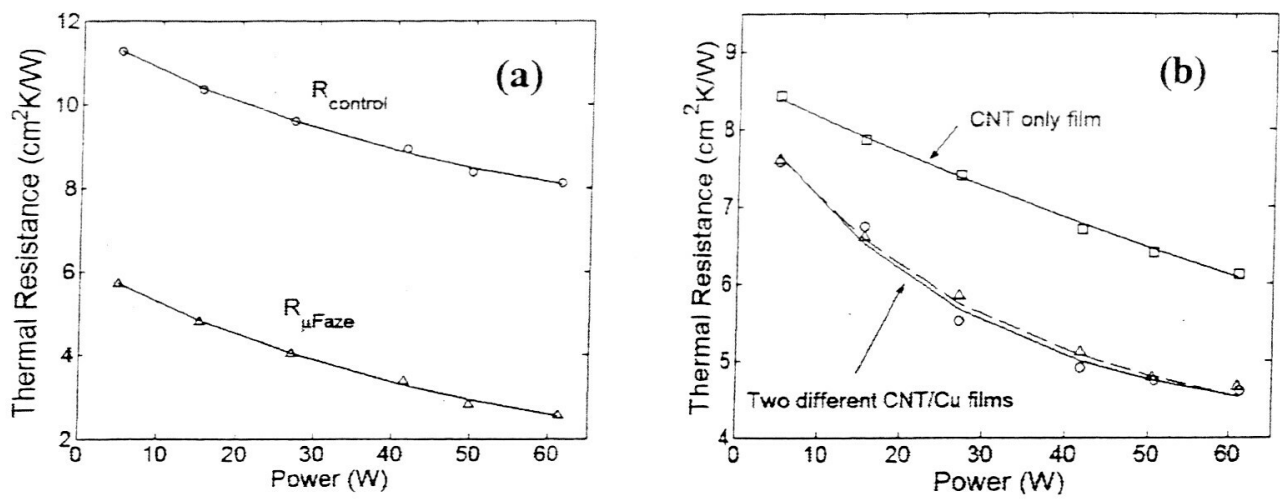

Figure 4. (a) Resistance versus power characteristics for first control sample and Microfaze. (b) Resistance versus power characteristics for CNT and CNT-Cu samples (before de-embedding Microfaze and upper copper block). The symbols represent measured data; the lines represent exponential fits to the data.

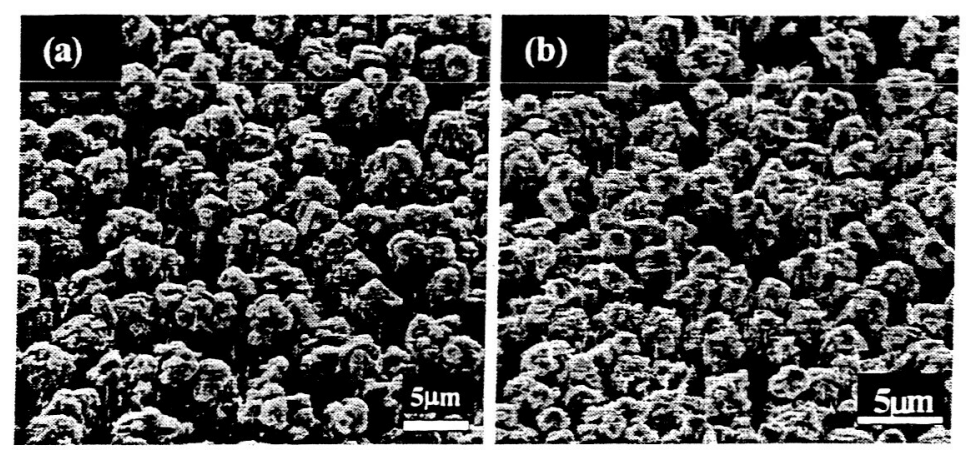

Figure 5. SEM micrographs (a) before and (b) after compressive thermal resistance measurement. The morphology of both the CNTs and $\mathrm{Cu}$ are similar in both cases.

\section{REFERENCES}

1. R. Viswanath, V. Wakharkar, A. Watwe, and V. Lebonheur, "Thermal Performance Challenges from Silicon to Systems", Intel Technology Journal, Q3 (2000).

2. P. Kim, L. Shi, A. Majumdar, and P.L. McEuen, "Thermal Transport Measurements of Individual Multiwalled Nanotubes", Phys. Rev. Lett., 87, p. $215502-1$ (2001).

3. B.A. Cruden, A.M. Cassell, Q. Ye, and M. Meyyappan, "Reactor Design Considerations in the Hot Filament/Direct Current Plasma Synthesis of Carbon Nanofibers", J. Appl. Phys, 94, 4070 (2003).

4. K. Kondo, N. Yamakawa, Z. Tanaka, and K. Hayashi, "Copper Damascene Electrodeposition and Additives", J. of Electroanalytical Chemistry, 559, 137 (2003).

5. H. Dai, J.H. Hafner, A.G. Rinzler, D.T. Colbert, and R.E. Smalley, "Nanotubes as Nanoprobes in Scanning Probe Microscopy", Nature, 384, 147 (1996).

6. H. Dai, N. Franklin, and J. Han, "Exploiting the Properties of Carbon Nanotubes for Nanolithography", Appl. Phys. Lett., 73, 1508 (1998).

7. J. Li, A.M. Cassell, and H. Dai, "Carbon Nanotubes as AFM Tips: Measuring DNA Molecules at the Liquid/Solid Interface", Surf. and Interf. Analysis, 28, 8 (1999) 